\title{
Functional Tests to Measure Bladder Wall Permeability for Assessing Bladder Pain Syndrome; A Mini Review
}

\author{
Rozenberg $\mathbf{B}^{1}$, Van Leuteren $\mathbf{R}^{2}$, Janssen $\mathbf{D}^{1}$ and Heesakkers $\mathbf{J}^{* 1}$ \\ ${ }^{1}$ Department of Urology, Radboud UMC Nijmegen, The Netherlands \\ ${ }^{2}$ Faculty of Technical Medicine, University of Twente, The Netherlands
}

Received: June 11, 2018; Published: June 20, 2018

*Corresponding author: John Heesakkers, Department of Urology, Radboud UMC Nijmegen, The Netherlands, Tel: 0031243616712 ;

Email: John.heesakkers@radboudumc.nl

\begin{abstract}
Abbreviations: BPS: Bladder Pain Syndrome; PST: Potassium Sensitivity Test; KCl: Potassium Chloride; $\mathrm{N}_{2} \mathrm{O}$ : Nitrous Oxide; SF: Fluorescein Sodium; TURP: Transurethral Resection of the Prostate
\end{abstract}

\section{Mini Review}

Bladder pain syndrome (BPS) is a chronic inflammatory status of the bladder wall with no known cause. Patients with BPS suffer from pain, pressure or inconvenience associated with the urinary bladder. Furthermore, urgency (increased feeling to urinate) or an increased frequency of micturition both during the day as well as during the night, has to be present [1]. Several studies have suggested an impaired barrier function, leading to increased bladder wall permeability of the urothelium as a likely cause for this disabling condition. In the healthy situation the bladder wall is impermeable to any substances within the urine. The urine is of an acidic composition and it is very important that the bladder wall serves as an important barrier to these acidic components. The best way to assess bladder wall permeability is still under debate. In this mini-review we compare the five different methods to determine bladder permeability.

\section{Permeability Measurement Methods}

Literature search with Medline, PubMED, Google Scholar, Scopus and the Cochrane library. Search terms such as 'interstitial cystitis', 'IC prevalence', 'Bladder pain syndrome', 'Hunner's lesion', 'bladder permeability', 'BPS diagnostics' and 'urothelium' were used to find related articles and after that more specific terms as 'fluorescence' and 'marker metabolization' were used. The search led to various articles investigating bladder permeability in vitro, in animals or in vivo. The procedures that described the permeability of the bladder were further investigated when at least three studies were found that investigated the method, in order to prevent possible positive or negative bias. The primary outcome of the studies had to be the result of a tested method to assess bladder wall permeability. Secondary outcome measures were the kind of situation the method was tested in (in vivo, in vitro), the medium of marker detection (urine, blood or exhaled breath) and the possible practical usability of the method. After the literature search, five measurement methods were selected to be evaluated.

\section{Potassium Test}

The potassium sensitivity test (PST) is the first test that offered a qualitative measure for bladder permeability, and was developed [2]. The test scores the increment in pain complaints of the patient after a potassium chloride (Kcl) $0.4 \mathrm{M}$ solution is administered in the bladder. Parsons stated that $75 \%$ of the BPS-patients had a worsening of pain complaints due to the potassium while healthy controls were barely sensitive to it. He investigated the accuracy of this method in BPS patients and $81 \%$ of the 244 patients had a positive test [3]. However, the evoked pain makes this test unpopular and subjective in clinical practice. As an improvement Daha used a lower concentration of $\mathrm{KCl}(0.2 \mathrm{M})$. The solution was used to investigate how the maximal bladder capacity varied between BPS patients and controls. Of the BPS patients $92 \%$ had a decreased maximal capacity of the bladder after the potassium instillation. The test could make the difference between BPS patients and controls and gave less pain that the original PST [4].

\section{Ethanol}

In order to find a less invasive method to measure bladder wall permeability, intravesical ethanol was investigated. For this an ethanol containing solution is instilled in the bladder. In order to derive a measure for bladder permeability the ethanol has been measured in exhaled breath. The more the ethanol is able to pass 
the urothelium, i.e. has a higher permeability, the higher the ethanol level in exhaled breath should be [5]. However, there was no detectable ethanol in the exhaled breath, therefore ethanol is an ineffective method to measure bladder permeability [6]. A possible explanation was that the breath analyzer is not sensitive enough for the low levels of ethanol. In support of the ethanol method Monga et al. performed a study in rabbits and found a reproducible and detectable amount of ethanol in the venous samples that were drawn from the animals in which the urothelium was damaged. However in humans no consistent results were found. Ethanol is an inexpensive substance that distributes evenly in the total body water [6]. However, ethanol is absorbed and metabolized by the liver, making the determination of ethanol as a clinical marker for this goal, less useful. The metabolization ensures that a lower ethanol level after the test is not entirely due to increased bladder wall permeability. The ethanol test's precision is too low in the current set-up, making it not suitable for permeability measurements.

\section{Laughing Gas}

Nitrous oxide $\left(\mathrm{N}_{2} \mathrm{O}\right)$ or laughing gas has been used since the nineteenth century and is used because of its anesthetic properties. In addition to its anesthetic use nitrous oxide has been tested as a permeability marker as well. Hahn studied tracer amounts of $\mathrm{N}_{2} \mathrm{O}$ in the exhaled breath of pigs in which a small concentration of $\mathrm{N}_{2} \mathrm{O}$ was administered either intravenously or intraperitoneally. He examined the usability of $\mathrm{N}_{2} \mathrm{O}$ as a possible marker for the absorption of irrigating fluids, during endoscopic interventions. The results showed a clear relation between the measured $\mathrm{N}_{2} \mathrm{O}$ in the exhaled breath and the boluses of irrigating fluid that were administered. This study showed that $\mathrm{N}_{2} \mathrm{O}$ functions according to the same principle as ethanol in order to detect volume absorption.

$\mathrm{N}_{2} \mathrm{O}$ is better than ethanol, but the volatility makes it hard to tell the cause of the concentration drop [7]. Piros included twelve healthy volunteers that received continuous or intermittent intravenous infusion of irrigation fluid. The results showed a proper estimation of the infused fluid that was absorbed, estimated with the N2O measurement in exhaled breath [8]. Drobin investigated the usability of $\mathrm{N} 2 \mathrm{O}$ in patients during a transurethral resection of the prostate (TURP). In this patient group the surface of the TURP lesion is suspect to absorb a large amount of fluids, leading to a TUR-syndrome that causes hyponatremia, nausea, dyspnea and abdominal pain [9]. These experiments state that perhaps laughing gas is suited to test bladder wall permeability.

\section{Technetium}

Radioactive technetium has also been suggested as a possible marker for bladder permeability. [10] Chelsky designed a study in which a small group of BPS-patients received a small amount of radioactive labeled technetium. This substance was later measured in a venous blood sample. It is a rather invasive method with a radiation load for the patients [10]. It was suggested as a very direct method for measuring the bladder permeability but only a limited amount of studies have been done with this method.

\section{Fluorescein}

This marker could use the intensity of fluorescence as a derivative for bladder permeability. Fluorescence is the emission of light of a certain wavelength by a substance that has absorbed light or electromagnetic radiation. The absorption of light excites electrons that move to a higher energetic shell around the molecule. When these electrons lose their high energetic state this behavior is associated with the emission of a photon, often as visible light. The emitted light has a longer wavelength than the absorbed light resulting in an excitation spectrum, the bandwidth of light that is absorbed, with a different color than the emission spectrum, the bandwidth of emitted light by the fluorophore. When the photon is emitted the cycle of excitation, transition and fluorescence can be passed again and again. Fluorescein sodium (SF) is used often in ophthalmology where it is used to diagnose pathologies of the cornea. Wood et al. performed a study in which the fluorescent characteristics were used to evaluate the assumed increase in bladder permeability. They used a Spectro fluorometer that measured the intensity of the fluorescence of a known concentration of fluorescein and pumped this solution into a bladder. After two minutes of circulation a sample was taken to determine the intensity of fluorescence. The loss in fluorescein intensity to predict the concentration make it plausible that the loss in fluorescein was absorbed by the bladder wall. However, this statement is poorly supported by the data and no firm conclusions can be drawn [11]. This idea of Wood is supported by a study of Eichel who investigated the bladder permeability using SF in mice. He instilled SF in the bladder and detected a delayed excretion of the substance when the bladder mucosa was injured, stating that this effect can be due to an increased permeability of the bladder caused by absorption [12]. Woodworth performed a study in humans in which SF was taken orally and an increased plasma concentration of SF and decreased excretion of SF was found [13]. These findings indicate a re-uptake of SF in the bladder.

\section{Conclusion}

Five methods have been tried and described in the literature to determine the urothelial leakage causing BPS by measuring bladder wall permeability. None of these methods is perfect and really applicable in the clinical setting. Therefore better tools have to be developed in order to test bladder wall permeability and to determine its role in the development of Bladder Pain Syndrome.

\section{References}

1. Merwe, J van der (2008) ESSIC Consensus on Criteria, Classification, and Nomenclature for PBS/IC, 1-2.

2. Parsons CL, Greenberger M, Gabal L, Bidair M, Barme G (1998) The Role of Urinary Potassium in the Pathogenesis And Diagnosis Of Interstitial Cystitis. J Urol 159(6): 1866-1867.

3. Parsons CL, Dell J, Stanford EJ, Bullen M, Kahn BS, et al. (2002) The prevalence of interstitial cystitis in gynecologic patients with pelvic pain, as detected by intravesical potassium sensitivity. Am J Obstet Gynecol 187(5): 1395-1400.

4. Daha L, Riedl C, Hohlbrugger G, Knoll M, Engelhardt PF, et al. (2003) Comparitive Assessment of Maximal Bladder Capacity, 0.9\% $\mathrm{NaCl}$ 
versus $0.2 \mathrm{M} \mathrm{Kcl}$, for the diagnosis of interstitial cystitis: a prospective controlled study. J Urol 170(3): 807-809.

5. Gordon Z, Parsons CL, Monga M (2003) Intravesical ethanol test: an ineffective measure of bladder hyperpermeability. Urology 61(3): 555557.

6. Monga M, Percival C,Zupkas P (2001) Intravesical Ethanol as Quantitative Measure of Bladder Hyperpermeability. J Endourol 15(6): 641-644.

7. Hahn RG (2003) Nitrous Oxide as a Marker for Irrigating Fluid Absorption. Scand. J Urol Nephrol 37(4): 281-285

8. Piros D, Drobin D, Hahn RG (2007) Nitrous oxide for monitoring fluid absorption in volunteers. Br J Anaesth 98(1): 53-59.

9. Drobin D, Hjelmqvist H, Piros D, Hahn RG (2008) Monitoring of fluid absorption with nitrous oxide during transurethral resection of the prostate. Acta Anaesthesiol Scand 52(4): 509-513.

10. Chelsky, S Rosen J (1994) Bladder permeability in interstitial cystitis is similar to that of normal volunteers: direct measurement by transvesical absorption of technetiumdethylenetriaminepetaacetic acid. J Urol 151(2): 346-349.

11. Wood R (2001) Human Bladder Permeability Assessment Using Intravesical Fluorescein Clearance Rate. J Urol :131-132.

12. Eichel L, Scheidweiler K, Kost J, et al. (2001) Fluorescein: Validation with Cyclophosphamide and Protamine. Urology 58(1): 113-118.

13. Woodworth BE, Buffington CA (1997) Excretion of Fluorescein in the Urine of Women With Interstitial Cystitis. J Urol 158(3): 786-789.

Assets of Publishing with us
BIOMEDICAL
RESEARCHES $\quad \begin{aligned} & \text { Global archiving of articles } \\ & \text { - Immediate, unrestricted online access }\end{aligned}$

